\title{
DÜBLIN
}

Technological University Dublin ARROW@TU Dublin

2009-06-01

\section{Inverse Scattering Solutions for Side-Band Signals}

\author{
Jonathan Blackledge \\ Technological University Dublin, jonathan.blackledge@tudublin.ie \\ Timo Hamalainen \\ University of Jyvaskyla, Finland, timo.t.hamalainen@jyu.fi \\ Jyrki Joutsensalo \\ University of Jyvaskyla, Finland, jyrki.j.joutsensalo@jyu.fi
}

Follow this and additional works at: https://arrow.tudublin.ie/engscheleart

Part of the Signal Processing Commons

\section{Recommended Citation}

Inverse Scattering Solutions for Side-Band Signals, Blackledge, Jonathan; ISSC 2009, UCD, June 10-11th 2009 , University College Dublin, 2009

This Conference Paper is brought to you for free and open access by the School of Electrical and Electronic Engineering at ARROW@TU Dublin. It has been accepted for inclusion in Conference papers by an authorized administrator of ARROW@TU Dublin. For more information, please contact arrow.admin@tudublin.ie, aisling.coyne@tudublin.ie,gerard.connolly@tudublin.ie.

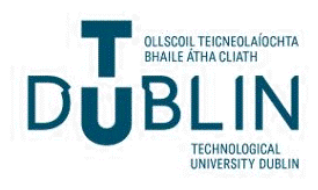




\title{
Inverse Scattering Solutions for Side-Band Signals
}

\author{
Jonathan Blackledge $^{*}$, Timo Hämäläinen ${ }^{\dagger}$ and Jyrki Joutsensalo ${ }^{\ddagger}$
}

SFI Stokes Professor of DSP
Dublin Institute of Technology
Ireland

E-mail: *jonathan.blackledge@dit.ie

${ }^{\dagger}$ timo.t.hamalainen@jyu.fi, ${ }^{\ddagger}$ jyrki.j.joutsensalo@jyu.fi

\author{
Department of Mathematical Information Technology \\ University of Jyväskylä \\ Finland

(1)


This equation requires an iterative procedure to be adopted in order determine $u_{s}$. However, under the weak field condition when $\left\|u_{s}\right\|<<\left\|u_{i}\right\|$ we consider the solution

$$
u_{s}(x, k)=k^{2} g(|x|, k) \otimes \gamma(x) u_{i}(x, k) .
$$

This is known as the Born approximation and has an asymptotic solution (for $x \rightarrow \infty$ and with $\left.u_{i}(x, k)=\exp (-i k x)\right)$ given by $[6]$

$$
u_{s}(x, k)=\frac{i k}{2} \exp (i k x) \int_{-\infty}^{\infty} \gamma(x) \exp (-2 i k x) d x .
$$

Thus, in the far field, the relationship between the scattering function and the scattered field is characterised by the Fourier transform, a relationship that is specific to the weak field condition. The scattering amplitude spectrum $A_{s}(k)$ (the 'reflection coefficient') can be defined as (with $k \rightarrow k / 2$ )

$$
A_{s}(k)=\frac{i k}{4} \widetilde{\gamma}(k)
$$

where

$$
\widetilde{\gamma}(k)=\int_{-\infty}^{\infty} \gamma(x) \exp (-i k x) d x
$$

and

$$
\gamma(x)=\frac{1}{2 \pi} \int_{-\infty}^{\infty} \widetilde{\gamma}(k) \exp (i k x) d k
$$

The purpose of this paper is to explore a solution for $\gamma$ and thereby $u_{s}$ when $\left\|u_{s}\right\| \sim\left\|u_{i}\right\|$ that is based on the result, from equation (1):

$$
\gamma(x)=-\frac{u^{*}}{|u(x, k)|^{2}}\left(1+\frac{1}{k^{2}} \frac{\partial^{2}}{\partial x^{2}}\right) u_{s}(x, k)
$$

\section{INVERSE SOLUTIONS}

Given equation (1), we can write

$$
\gamma(x)=-\frac{1}{k^{2} u(x, k)}\left(k^{2}+\frac{\partial^{2}}{\partial x^{2}}\right) u(x, k)
$$

which, noting that $\gamma=\epsilon_{r}-1$ can be written in the form

$$
\epsilon_{r}(x)=-\frac{u^{*}(x, k)}{k^{2}|u(x, k)|^{2}} \frac{\partial^{2}}{\partial x^{2}} u(x, k)
$$

and since $u=u_{i}+u_{s}$ we can write

$$
\begin{aligned}
\gamma(x) & =-\frac{u_{i}^{*}(x, k)+u_{s}^{*}(x, k)}{k^{2}\left|u_{i}(x, k)+u_{s}(x, k)\right|^{2}} \\
& \times\left(k^{2}+\frac{\partial^{2}}{\partial x^{2}}\right) u_{s}(x, k) .
\end{aligned}
$$

Note that when $\left\|u_{s}\right\|<<\left\|u_{i}\right\|$,

$$
\left(\frac{\partial^{2}}{\partial x^{2}}+k^{2}\right) u_{s}(x, k)=-k^{2} \gamma(x) u_{i}(x, k),
$$

the equivalent expression for $\gamma(x)$ being

$$
\begin{aligned}
\gamma(x) & =-\frac{u_{i}^{*}(x, k)}{k^{2}\left|u_{i}(x, k)\right|^{2}}\left(k^{2}+\frac{\partial^{2}}{\partial x^{2}}\right) u_{s}(x, k) \\
& =-\frac{u_{i}^{*}(x, k)}{k^{2}}\left(k^{2}+\frac{\partial^{2}}{\partial x^{2}}\right) u_{s}(x, k)
\end{aligned}
$$

given that $u_{i}(x, k)=\exp ( \pm i k x)$ is a solution of equation (2). In comparison with the solution under the Born approximation, this solution for $\gamma$ includes $|u(x, k)|^{-2}$ which describes the (inverse square) amplitude envelop of the sum of the incident and scattered fields, i.e. with $u(x, k)=$ $A_{u}(x, k) \exp \left[i \theta_{u}(x, k)\right]$, it is clear that $|u(x, k)|^{2}=$ $\left[A_{u}(x, k)\right]^{2}$.

\section{a) Solution for Side-band Signals}

If $u$ is taken to be a narrow side-band signal that is dominated by a high frequency and carrier wave characterised by $k_{0}$, which, in turn, is determined by a narrow side-band incident wavefield $u_{i}$, then we can consider the condition where $\left|u\left(x, k_{0}\right)\right|^{2} \sim$ $1 \forall x$ (in general, a constant). This condition is equivalent to considering the case where $u$ is a phase only field $u\left(x, k_{0}\right)=\exp \left[i \theta_{u}\left(x, k_{0}\right)\right]$ and is imposed in respect of the fact that, for narrow side-band signals, the contribution of $|u|^{-2}$ to the reconstruction of $\gamma$ from $u$ is not significant when compared to $\left(u_{i}^{*}+u_{s}^{*}\right)\left(k_{0}^{2}+\partial_{x}^{2}\right) u_{s}$. However, strictly speaking, the condition being imposed implies that, with $u_{i}=\exp \left( \pm i k_{0} x\right)$,

$$
u_{i}^{*} u_{s}+\left(u_{i}^{*} u_{s}\right)^{*}+\left|u_{s}\right|^{2}=0 .
$$

The principal difference between the reconstruction of $\gamma$ using the Born approximation and the inverse solution considered here is compounded in the addition of a single term, i.e. for a weak field where $\left\|u_{s}\right\|<<\left\|u_{i}\right\|$

$$
\gamma(x)=-u_{i}^{*}\left(x, k_{0}\right)\left(1+\frac{1}{k_{0}^{2}} \frac{\partial^{2}}{\partial x^{2}}\right) u_{s}\left(x, k_{0}\right)
$$

and for a strong field where $\left\|u_{s}\right\| \sim\left\|u_{i}\right\|$

$$
\begin{gathered}
\gamma(x)=-u_{i}^{*}\left(x, k_{0}\right)\left(1+\frac{1}{k_{0}^{2}} \frac{\partial^{2}}{\partial x^{2}}\right) u_{s}\left(x, k_{0}\right) \\
-u_{s}^{*}\left(x, k_{0}\right)\left(1+\frac{1}{k_{0}^{2}} \frac{\partial^{2}}{\partial x^{2}}\right) u_{s}\left(x, k_{0}\right) .
\end{gathered}
$$

Note that under the strict definition of a phase only field which leads to equation (3), for $\left\|u_{s}\right\| \sim$ $\left\|u_{i}\right\|$

$$
\begin{gathered}
\gamma(x)=u_{i}\left(x, k_{0}\right) u_{s}^{*}\left(x, k_{0}\right) \\
-\frac{\left[u_{i}\left(x, k_{0}\right)+u_{s}\left(x, k_{0}\right)\right]^{*}}{k_{0}^{2}} \frac{\partial^{2}}{\partial x^{2}} u_{s}\left(x, k_{0}\right) \\
=-u_{i}\left(x, k_{0}\right) u_{s}^{*}\left(x, k_{0}\right), \quad k_{0} \rightarrow \infty
\end{gathered}
$$

In this paper, we relax this condition and utilze equations (4) and (5) given that $\left|u_{i}+u_{s}\right|^{2} \sim 1$. This is the 'key' to the results that follow. 


\section{b) Numerical Simulation}

We consider a numerical simulation based on computing the scattered field using a forward differencing approach to equation (1). For a fixed wavenumber $k_{0}$ (which defines a continuous wave mode), the vector $u_{n}$ computed over a uniformly sampled discrete array composed of $N$ samples, each of length $\Delta$, is given by

$\frac{u_{n+1}-2 u_{n}+u_{n-1}}{\Delta^{2}}+k_{0}^{2} u_{n}=-k_{0}^{2} \gamma_{n} u_{n}, \quad n \in[1, N]$

which has the solution

$$
u_{n}=\frac{u_{n+1}+u_{n-1}}{2-\Delta^{2} k_{0}^{2}\left(1+\gamma_{n}\right)}=\frac{u_{n+1}+u_{n-1}}{2-\Delta^{2} k_{0}^{2} \epsilon_{r, n}} .
$$

A solution to equation (6) can be obtained that is based on the following iteration:

$$
u_{n}^{m+1}=\frac{u_{n+1}^{m}+u_{n-1}^{m}}{2-\Delta^{2} k_{0}^{2} \epsilon_{r, n}}
$$

where $u_{n}^{1}$ is taken to be a discrete representation of the (unit amplitude) incident field which we define with a fixed number of periods $p$ over $n \in[1, N]$, i.e.

$$
u_{n}^{1}=\exp \left(\frac{2 \pi i p(n-1)}{(N-1)}\right) .
$$

A necessery condition that must be applied to equation (7) is that

$$
\Delta k_{0}<\sqrt{\frac{2}{\left\|\epsilon_{r, n}\right\|_{\infty}}}
$$

where $\|\bullet\|_{\infty}$ defines the 'uniform norm' and

$$
k_{0}=\frac{2 \pi p}{N} .
$$

Since a solution to the equation (1) is not conditional on the amplitude of the wavefield (i.e. the incident field can be $A \exp \left( \pm i k_{0} x\right)$ where $A$ is an arbitrary value), the amplitude of the array $u_{n}^{m}$, after $M$ iterations is normalised on output, i.e. $u_{n}^{M} \rightarrow u_{n}^{M} /\left\|u_{n}^{M}\right\|_{\infty}$.

For the discrete case, equations (4) and (5) transform to (for $M$ iterations used to compute the scattered field)

$$
\begin{aligned}
& \epsilon_{r, n}=1-u_{i, n}^{*}\left[u_{s, n}+\left(\Delta k_{0}\right)^{-2} u_{s, n} \otimes(1,-2,1)\right] \\
= & 1-\left(u_{n}^{1}\right)^{*}\left[\left(u_{n}^{M}-u_{n}^{1}\right)+\left(\Delta k_{0}\right)^{-2}\left(u_{n}^{M}-u_{n}^{1}\right) \otimes(1,-2,1)\right]
\end{aligned}
$$

and

$$
\begin{gathered}
\epsilon_{r, n}=1-u_{n}^{*}\left[u_{s, n}+\left(\Delta k_{0}\right)^{-2} u_{s, n} \otimes(1,-2,1)\right] \\
=1-\left(u_{n}^{M}\right)^{*}\left[\left(u_{n}^{M}-u_{n}^{1}\right)+\left(\Delta k_{0}\right)^{-2}\left(u_{n}^{M}-u_{n}^{1}\right) \otimes(1,-2,1)\right]
\end{gathered}
$$

respectively where $\otimes$ now denotes the discrete convolution sum. Figures 1-2 show comparisons between the numerical results given by equations (8) and (9), illustrating the superiority of the inverse scattering solution considered over that given by the weak field solution for the case when $k_{0} \Delta=0.01$. These results are based on applying the initial solution $u_{n}^{1}=\sin [2 \pi i p(n-1) /(N-1)]$ and Hilbert transforming the output arrays before computation of equations (8) and (9) through use of the MATLAB function hilbert. The profile of the reconstruction for $\epsilon_{r, n}$ based on equation (9) is preserved inclusive of characteristic 'ringing' due to the discontinuities associated with the model for $\epsilon_{r, n}$. In comparison, the weak field solution given by equation (8) has a relatively narrow dynamic range and provides a poor reconstruction particularly with regard to resolving the discontinuities associated with $\epsilon_{r, n}$.

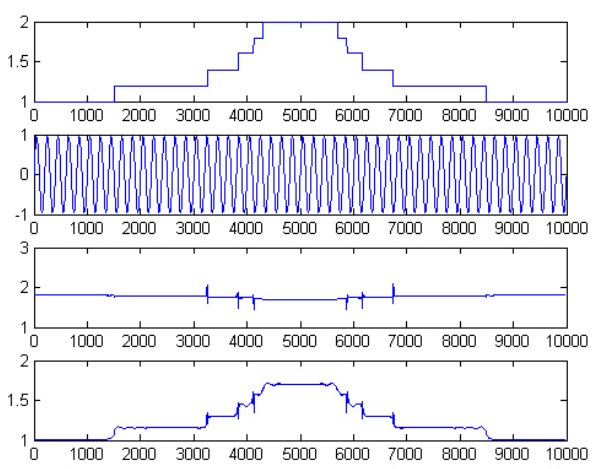

Fig. 1: Comparison between the weak and strong field inverse scattering solutions for the case when $N=10000$, $M=100, \Delta k_{0}=0.01$ with $p=50$. From top to bottom: Relative permittivity function model $\epsilon_{r, n}$; real part of wavefield computed via equation (7); inverse solution (real part) computed using equation (8); inverse scattering solution (real part) computed using equation (9).

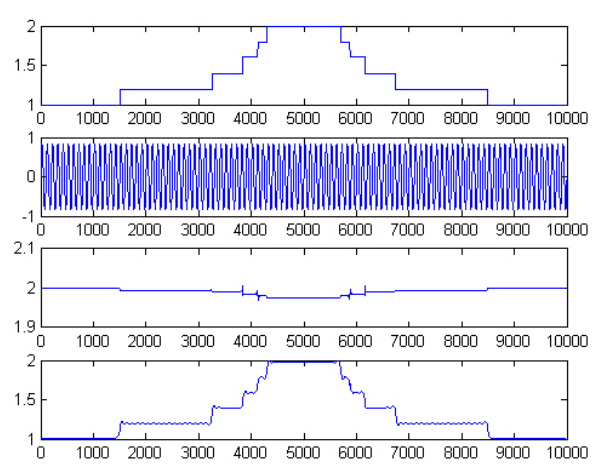

Fig. 2: Comparison between the weak and strong field solutions for the case when $N=10000, M=100, \Delta k_{0}=0.01$ with $p=100$. The descriptions of each plot follow those as given in Figure 1. 


\section{Solution for Narrow Side-BAnd Pulse-ECho Systems}

The inverse solutions derived and demonstrated numerically in the previous section have little practical value with regard to engineering a system designed to recover $\epsilon_{r}(x)$ from information on the scattered field measured 'outside' the scatterer, i.e. when $|x|>|X|$. This is because the solutions considered require that the scattered field is known $\forall x$ including $\forall x \in[-X, X]$. Practically applicable systems typically measure the scattered field at a fixed point $x$ in the far field (i.e. as $x \rightarrow \infty$ ) by recording the spectrum of $u_{s}$ over a range of values of $k$. In practice, this requires the application of pulse-echo mode methods.

Pulse-echo methods involve the emission of a pulse and a recording of the back-scattered wavefield (echo). This approach is consistent with the physical nature a system if: (i) the scattering function is a one-dimensional function; (ii) the incident wavefield is a 'pencil-line beam'. However, since all physical systems are intrinsically threedimensional, this model is idealised. Nevertheless, a variety of electromagnetic information and imaging systems can be 'cast' in terms of problems involving layered materials (e.g. the response of light, radio and microwaves to layered dielectric materials including the propagation of electromagnetic waves along transmission lines such as an optical fiber).

For a narrow side-band system with carrier frequency $k_{0}>>|k|$, the inverse scattering solution is given by (for $\left|A_{u}\right| \sim 1$ )

$$
\gamma(x)=-\frac{u^{*}(x, k)}{k_{0}^{2}}\left(k_{0}^{2}+\frac{\partial^{2}}{\partial x^{2}}\right) u_{s}(x, k)
$$

which, for a weak field where $\left\|u_{s}\right\|<<\left\|u_{i}\right\|$ reduces to

$$
\gamma(x)=-\frac{u_{i}^{*}(x, k)}{k_{0}^{2}}\left(k_{0}^{2}+\frac{\partial^{2}}{\partial x^{2}}\right) u_{s}(x, k) .
$$

These results apply for all values of $x \in(-\infty, \infty)$. As discussed in Section I, under the Born approximation, for $x \rightarrow \infty$, a mapping is obtained between the scattered field $u_{s}$ and $\gamma$ that is based on the Fourier transform $\widetilde{\gamma}(k)$ of $\gamma(x)$, i.e.

$$
u_{s}(x, k)=\frac{i k_{0}}{2} \exp (i k x) \widetilde{\gamma}(k), \quad|k|<<k_{0}
$$

which is a solution of

$$
\gamma(x)=-\frac{u_{i}^{*}(x, k)}{k_{0}^{2}}\left(k_{0}^{2}+\frac{\partial^{2}}{\partial x^{2}}\right) u_{s}(x, k) .
$$

This result suggests taking the Fourier transform of the equation

$$
\gamma(x)=
$$

$$
-\left[u_{i}^{*}\left(x, k_{0}\right)+u_{s}^{*}\left(x, k_{0}\right)\right]\left(1+\frac{1}{k_{0}^{2}} \frac{\partial^{2}}{\partial x^{2}}\right) u_{s}\left(x, k_{0}\right)
$$

giving

$$
\begin{gathered}
\widetilde{\gamma}(k)= \\
-\left[\widetilde{u}_{i}^{*}\left(k, k_{0}\right)+\widetilde{u}_{s}^{*}\left(k, k_{0}\right)\right] \odot\left(1-\frac{|k|^{2}}{k_{0}^{2}}\right) \widetilde{u}_{s}\left(k, k_{0}\right) \\
=-\left[\widetilde{u}_{i}^{*}\left(k, k_{0}\right)+\widetilde{u}_{s}^{*}\left(k, k_{0}\right)\right] \odot \widetilde{u}_{s}(k), \quad|k|<<k_{0}
\end{gathered}
$$

where $\widetilde{\gamma}, \widetilde{u}_{i}^{*}, \widetilde{u}_{s}^{*}$ and $\widetilde{u}_{s}$ are the Fourier transforms of $\gamma, u_{i}^{*}, u_{s}^{*}$ and $u_{s}$ respectively and $\odot$ denotes the correlation integral. Noting that, for $u_{i}\left(x, k_{0}\right)=$ $\exp \left(-i k_{0} x\right), \widetilde{u}_{i}^{*}\left(k, k_{0}\right)=2 \pi \delta\left(k_{0}-k\right)$ we have

$\widetilde{\gamma}(k)=2 \pi \widetilde{u}_{s}\left(k-k_{0}\right)-\widetilde{u}_{s}^{*}(k) \odot \widetilde{u}_{s}(k), \quad|k|<<k_{0}$

or (ignoring scaing by $2 \pi$ )

$$
\widetilde{u}_{s}(k)=
$$

$\widetilde{p}(k) \widetilde{\gamma}\left(k+k_{0}\right)+\widetilde{p}(k)\left[\widetilde{u}_{s}^{*}\left(k+k_{0}\right) \odot \widetilde{u}_{s}\left(k+k_{0}\right)\right], \quad \forall k$

where $\widetilde{p}(k)$ is some lowpass filter with a bandwidth significantly less than $k_{0}$. Thus, upon takling the inverse Fourier transform, we obtain an expression for the (demodulated) scattered field $u_{s}$ given by

$$
\begin{gathered}
u_{s}\left(x, k_{0}\right)=p(x) \otimes\left[\gamma(x) \exp \left(-i k_{0} x\right)\right] \\
+p(x) \otimes\left[\left|u_{s}\left(x, k_{0}\right)\right|^{2} \exp \left(-i k_{0} x\right)\right]
\end{gathered}
$$

where $p(x)$ is the inverse Fourier transform of $\widetilde{p}(k)$. Here, $p(x)$ described the bandlimited pulse that is incident on a layered dielectric described by $\gamma(x)$. The first term is a description for the weak scattered field and the second term describes the effects of multiple scattering. In terms of the 'standard model' for a stationary signal $s(t)$ as a function of time $t$ where

$$
s(t)=p(t) \otimes f(t)+n(t)
$$

it is now clear that the Impulse Response Function (IRF) $f$ is given by

$$
f(t)=\gamma(t) \exp \left(-i \omega_{0} t\right)
$$

and the noise $n(t)$ is given by

$$
n(t)=p(t) \otimes\left[|s(t)|^{2} \exp \left(-i \omega_{0} t\right)\right]
$$

where $\omega_{0}$ is the angular (carrier) frequency. Note that in practice, $n(t)$ will include additional background noise and in this sense, we have extract a component of the noise term that is attributed to multiple scattering effects, albeit under the conditions that: (i) $\left|u_{i}+u_{s}\right|^{-2} \sim 1$; (ii) $|k|<<k_{0}$. 


\section{Linear FM Signal Processing}

A linear FM 'chirp' signal, which is taken to be of compact support $t \in[-T / 2, T / 2]$, is given by (in complex form and for a unit amplitude)

$$
p(t)=\exp \left(i \alpha t^{2}\right), \quad|t| \leq \frac{T}{2}
$$

where $\alpha$ is a constant which defines the 'chirp rate' and $T$ is the length of the 'pulse'. The phase of this signal is given by $\alpha t^{2}$ (i.e. it has a quadratic phase factor) and its instantaneous frequency is therefore given by $2 \alpha t$ which varies linearly with time $t$. The frequency modulations are linear, the signal therefore being referred to as a 'linear' FM pulse.

\section{a) Inversion under the Weak Field Condition}

Consider a signal generated by the reflection of a linear FM pulse. Application of the weak field condition yields the following single scattering model:

$$
s(t)=\exp \left(i \alpha t^{2}\right) \otimes f(t), \quad|t| \leq \frac{T}{2} .
$$

Using a pulse of this type provides a simple but effective way of recovering the IRF by correlating the signal with the complex conjugate of the pulse to give the reconstruction

$$
\begin{aligned}
\hat{f}(t)= & \exp \left(-i \alpha t^{2}\right) \odot \exp \left(i \alpha t^{2}\right) \otimes f(t), \quad|t| \leq \frac{T}{2} \\
& =T \exp \left(-i \alpha t^{2}\right) \operatorname{sinc}(\alpha T t) \otimes f(t) \\
& \simeq T \operatorname{sinc}(\alpha T t) \otimes f(t), \quad T>>1 .
\end{aligned}
$$

This simplification, under the condition that $T>>$ 1 , is not only practically applicable, but systems desirable, especially with regard to electromagnetic pulse generation ${ }^{1}$ and allows the result for $\hat{f}$ to be easily analysed in Fourier space. Using the convolution theorem we can write (ignoring scaling by $\pi / \alpha)$

$$
\hat{F}(\omega)= \begin{cases}F(\omega), & |\omega| \leq \alpha T \\ 0, & |\omega|>\alpha T .\end{cases}
$$

which describes $\hat{f}$ as being a band-limited version of $f$ (given that $f$ is not a band-limited function) where the bandwidth is determined by $\alpha T$.

\section{b) Inversion Under the Strong Field Condition}

For a side-band FM system, the results discussed above imply that, under the single scattering approximation

$$
s(t)=p(t) \otimes f(t)
$$

\footnotetext{
${ }^{1} \mathrm{~A}$ 'long pulse' provides greater signal energy and thus, the recorded data has a higher Signal-to-Noise Ratio.
}

with inverse scattering estimate

$$
\hat{f}(t)=p^{*}(t) \odot s(t)
$$

where, for a side-band system $p(t)=\exp \left(i \alpha t^{2}\right)$. Here, $s(t)$ is taken to be the (complex) analytic signal obtained by taking the Hilbert transform of the real signal $\operatorname{Re}[s(t)]$. For side-band systems this is typically undertaken by quadrature detection during demodulation from side-band to base-band [5].

The strong field inverse scattering solution for the signal, given by equation (10), implies that for the same (side-band) system

$$
\begin{gathered}
\hat{f}(t)=T \operatorname{sinc}(\alpha T t) \otimes f(t) \\
+T \operatorname{sinc}(\alpha T t) \otimes\left[|s(t)|^{2} \exp \left(-i \omega_{0} t\right)\right] .
\end{gathered}
$$

\section{Applications to Synthetic Aperture RADAR}

Synthetic Aperture Radar (SAR) is a side-band pulse-echo system which utilizes the response of a scatterer as it passes through the radar beam to synthesize the lateral (azimuth) resolution [8], [9]. This allows relatively high resolution images to be obtained at a long range. The antenna emits a pulse of microwave radiation toward the ground and the return signal is recorded at fixed time intervals along the flight path.

By studying the response of a SAR to a point scatterer in range $x$, and then in azimuth $y$, the Point Spread Function of the system is established which is given by $[7]$

$$
p(x, y)=\operatorname{sinc}(\alpha L x) \operatorname{sinc}\left(\beta k_{0} y\right)
$$

where $L$ is the pulse length and $\beta$ is the angle of divergence of the radar beam. Thus, the (postprocessed) SAR image data $\hat{f}(x, y)$ is given by the convolution of the object function $f(x, y)$ (e.g. the 'ground truth') with the appropriate point spread function, i.e.

$$
\hat{f}(x, y)=p(x, y) \otimes_{2} f(x, y)
$$

where $\otimes_{2}$ denotes the two-dimensional convolution integral and $\hat{f}$ is taken to be complex data generated by $f$. A SAR image is usually generated by displaying the amplitude modulations of the data, i.e.

$$
I_{S A R}(x, y)=|\hat{f}(x, y)| .
$$

The object function describes the imaged properties of the ground or sea surface. A conventional model for this function is the point scattering model that is typically used in SAR simulations [10]. This is where the object function is taken to be a distribution of point scatterers each of which reflects a replica of the emitted pulse and responds identically in azimuth. Here, nothing is said about the true physical nature of the 
object function in terms of material (dielectric) properties. Moreover, this standard convolution model for the data is based on application weak field approximation where multiple scattering effects (which generate 'speckle') are taken to be part of an additive noise function [7]. SAR images are coherent images (i.e. based on complex data containing magnitude and phase information) and consequently, contain noise that is characteristic of speckle patterns (coherent noise).

Based on equation (11), we consider a model where

$\hat{f}(x, y)=p(x, y) \otimes_{2}\left[f(x, y)+|s(x, y)|^{2} \exp \left(-i k_{0} x\right)\right]$

where the second term (on the right hand side) is taken to be the multiple scattering term ${ }^{2}$. Figure 3 shows the effect of applying a Gaussian lowpass filter to the complex data $\hat{f}(x, y)$ before generation of the image $|\hat{f}(x, y)|$ using data obtained from the Sandia National Laboratories SAR database [11]. Filtering the complex data is undertaken in order to attempt to suppress the term $p(x, y) \otimes_{2}[\mid$ $\left.\left.s(x, y)\right|^{2} \exp \left(-i k_{0} x\right)\right]$. The more usual practice is to filter the amplitude image $|\hat{f}|$. However, within the context of equation (12), applying a lowpass filter prior to computing an amplitude image of the complex data reduces the influence of the cross terms inherent in $|\hat{f}(x, y)|$ and it is this effect that reduces the speckle as illustrated in Figure 3.
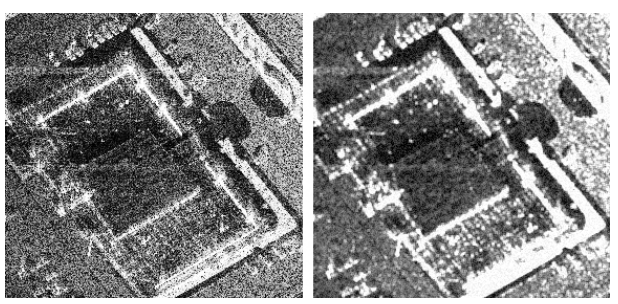

Fig. 3: Example of an original SAR image (left) and the same image after lowpass filtering the complex data (right). In both cases, the images have been histogram equalized utilizing the MATLAB function histeq.

\section{CONCLUSION}

The extension of the standard (stationary) model $s(t)=p(t) \otimes f(t)$ to include multiple scattering effects that are compounded in a single additional term provides a practical method of processing signals that is compatible with a standard signal processing 'toolkit'. The inverse scattering problem is usually formulated by first solving the forward scattering problem. Once the relationship between the scattered field and the scattering function has been established by solving the Helmholtz equation, an inverse solution is then attempted. The approach taken in this paper has

\footnotetext{
${ }^{2}$ After application of conventional SAR signal processing.
}

been to work directly with the Helmholtz equation to produce an expression for the scattering function. The example numerical simulations given in Section II(b) provide evidence of the expected superiority of this solution over the weak scattering solution. However, it should be noted that this result is based on the application of the narrow side-band condition which is required in order to set $\left|u_{i}(x, k)+u_{s}(x, k)\right|^{2} \sim 1$. In this sense, the solution developed is not based on an entirely 'exact' inverse scattering solution, but rather a modified version of a solution tailored for applicability to narrow side-band systems. Such systems are consistent with the applications of electromagnetic signal processing.

A further extension of the inverse scattering approach considered here that is independent of the condition $\left|u_{i}(x, k)+u_{s}(x, k)\right|^{-2} \sim 1$ can be developed using the expansion

$\left|u_{i}(x, k)+u_{s}(x, k)\right|^{-2}=1-u_{i} u_{s}^{*}-u_{i}^{*} u_{s}-\left|u_{s}\right|^{2}+\ldots$

details of which lie beyond the scope of this paper.

\section{REFERENCES}

[1] Z. S. Agranovich and V. A. Marchenko, "The Inverse Problem of Scattering Theory", Gordon and Breach, 1963.

[2] K. Chandan and P. C. Sabatier, "Inverse Problems in Quantum Scattering Theory", Springer, 1977.

[3] M. Cheney, J. H. Rose and B. DeFacio "Three Dimensional Inverse Scattering", Differential Equations and Mathematical Physics, Springer, 46-54, 1987.

[4] P. M. Morse and H. Feshbach, "Methods of Theoretical Physics", McGraw-Hill, 1953.

[5] J. M. Blackledge, "Digital Signal Processing", Horwood Publishing, 2006.

[6] F, J. W. Olver, "Asymptotics and Special Functions", Academic Press, 1974.

[7] J. M. Blackledge, "Digital Image Processing", Horwood Publishing, 2006.

[8] R. O. Harger, "Synthetic Aperture Radar Systems", Academic Press, 1970.

[9] J. J. Kovaly, "Synthetic Aperture Radar", Artech, 1976.

[10] R, L. Mitchell, "Radar Signal Simulation", MARK Resources, 1985.

[11] http://www.sandia.gov/RADAR/sardata.html 